\title{
Acceptability, feasibility and perceived satisfaction of the use of the Atraumatic Restorative Treatment approach for people with disability
}

\section{Gustavo Fabián MOLINA(a) Denise FAULKS ${ }^{(b)}$ Joannes FRENCKEN(c)}

(a) Universidad Nacional de Córdoba - UNC, Facultad de Odontología, Department of Dental Materials, Córdoba, Argentina.

(b)Université d'Auvergne - UdA, Service d'Odontologie, CHU Clermont-Ferrand, Clermont Ferrand, France.

(c)Radboud University Medical Centre, College of Dental Sciences, Department of Global Oral Health, Radboud, Nijmegen, The Netherlands.

Declaration of Interests: The authors certify that they have no commercial or associative interest that represents a conflict of interest in connection with the manuscript.

Corresponding Author:

Gustavo Fabián Molina

E-mail:gfmolina@hotmail.com

DOI: 10.1590/1807-3107BOR-2015.vol29.0097

Submitted: Nov 24, 2014

Accepted for publication: May 04, 2015

Last revision: Jul 02, 2015

\begin{abstract}
Unmet caries treatment need is prevalent among people with disability, partly due to difficulties cooperating with conventional dental treatment. This study compared Atraumatic Restorative Treatment (ART) with conventional restorative treatment (CRT) in the clinic and under general anaesthesia (GA), in terms of feasibility, acceptability and respondent satisfaction in patients referred for special care dentistry. Patients referred for dental restorative care were treated using either ART or CRT approach. Acceptance, feasibility and level of satisfaction with the treatment provided were assessed. ANOVA with Bonferroni correction and Chi-square tests investigated differences in age, gender and Visual Analogue Scale satisfaction scores. A total of 66 patients (mean $13.6 \pm 7.8$ years) were included and 43 respondents chose ART. ART was feasible for 47 patients, with optimal placement of restorations for $79 \%$ of all patients receiving ART. CRT in the clinic was chosen by 15 respondents and was feasible for 5 (33\%). Local anaesthesia was required for 4 of the 47 patients receiving ART and for 3 of the 5 patients receiving CRT in the clinic. Neither ART nor CRT could be performed in the clinic for 14 patients who were treated under GA (21\%). Respondent satisfaction was higher for those receiving ART than CRT (in the clinic and under GA). It was concluded that ART is a satisfactory, feasible, acceptable and effective approach to restorative dental treatment in patients with disability who have difficulty coping with conventional treatment. More research is now required to confirm these results in a larger study population.
\end{abstract}

Keywords: Dental Atraumatic Restorative Treatment; Dental Care for Disabled; Professional Practice; Intellectual Disability.

\section{Introduction}

Accessibility to dental care for people with disability is an issue worldwide and is particularly problematic in developing countries today., Unfortunately, many countries still lack the policies, systems and services required to improve access. Dental treatment for the disadvantaged most often depends upon the local efforts of benevolent practitioners or non-governmental organisations. Even in the rare countries where 
dedicated dental services for special needs patients do exist, environmental and societal barriers may prevent optimal use of services ${ }^{3,4}$

When the problem of access to appropriate services is aligned with data from the numerous oral epidemiological surveys concerning people with disability, it is clear that this population is subject to discrimination and inequality in oral health. ${ }^{1,2}$ A recent systematic review revealed an equal to lower prevalence of caries in adults with intellectual disabilities but a higher prevalence of periodontal disease compared to the general population. ${ }^{5}$ The major differences for the group with disability were a higher number of untreated carious lesions, lack of oral care and infrequent use of preventive strategies. In terms of restorative dentistry, it is particularly interesting to investigate the barriers to the placement of restorations in this population.

The provision of high quality restorative treatment is related to the patient's ability to cope with the anxiety engendered by treatment and to cooperate fully with the demands of the clinical situation. ${ }^{6,7}$ Between a quarter and a third of adults with intellectual disability are estimated to have dental anxiety. ${ }^{8,9,10}$ Unpleasant stimuli, such as the injection of local anaesthesia, or the noise and vibration of rotary instruments, may provoke disproportionate anxiety and subsequent opposition to treatment both in the short and long term. ${ }^{11,12}$ In addition, poor muscle coordination, fatigability, and oral dysfunction, such as drooling and tongue movement, may compromise restorative procedures. The use of moisture sensitive restorative materials may be particularly problematic. ${ }^{13}$ Sedation or general anaesthesia may improve clinical conditions for restorative work ${ }^{14}$ but these techniques have their own problems in terms of accessibility, cost and patient morbidity and mortality. ${ }^{15}$

A recently published systematic review concluded that no uniform preventive or restorative treatment programme could be endorsed for persons with disability. Reported restorative treatment protocols included the use of high-viscosity glass-ionomer and resin-modified glass-ionomer in addition to the use of a chemo-mechanical caries removal gel. ${ }^{16}$ The review findings showed that caries management in people with disability seems to be unstructured and that evidence for a specific preventive and/or treatment programmes is not yet available. This review, amongst others, suggested that the Atraumatic Restorative Treatment approach (ART) might help to reduce barriers to restorative care for patients with disabilities ${ }^{16,17}$ but no clinical trial has yet been reported that tests this theory.

ART is a non-invasive approach to restorative treatment that is well-recognised, has a solid evidence base accumulated over the last 25 years, and is endorsed by the World Health Organisation. ${ }^{18}$ ART involves hand instrumentation and placement of high-viscosity glass-ionomer cement restorations. The potential advantages of ART in the population with disability are that the drill is avoided and the needle is rarely used, and therefore the approach is less likely to provoke severe anxiety. ${ }^{18,19}$ In 2012, a survey amongst experts in special care dentistry was undertaken to collect opinion regarding the ART approach for caries treatment in people with disability. All respondents reported having full or moderate knowledge of ART $(23.3 \%$ and $63.3 \%$ respectively), and $66.7 \%$ indicated that the approach was useful for this population. However, only $50 \%$ of respondents used the approach regularly in their practice. ${ }^{20}$ One of the barriers cited in the survey was lack of scientific evidence supporting the use of ART specifically in the population with disability. Therefore, a prospective clinical trial was set up amongst people with a disability with the aim to investigate the survival of ART restorations and the satisfaction, acceptability and feasibility of ART in comparison to conventional restorative care. The survival rates have been recently published and showed a $98 \%$ survival of all ART high-viscosity glass-ionomer restorations and a $91 \%$ survival of all conventional composite restorations after one year. ${ }^{21}$

The present study, the first in its kind, is aimed to compare ART with conventional restorative treatment in terms of acceptability and feasibility of the approach and respondent satisfaction in a population of patients referred for special care restorative dentistry. 


\section{Methodology \\ Ethics}

Ethical approval was obtained from the local Ethical Committee, CIEIS Facultad de Odontología, Universidad Nacional de Córdoba with the reference number 38/2012 and the trial was registered at Netherlands Trial Register with number 4400. As a detailed report about the methodology used is presented elsewhere, ${ }^{21}$ a shortened version is presented underneath.

\section{Participants}

All patients with a disability referred for restorative treatment to a dental hospital clinic over a six months period (Aug 2012 to Feb 2013) were considered for inclusion in the study. Patients were examined by one of two special care dentists. Medical history was taken. A full description of the functional, social and environmental context of the patient was recorded using the International Classification of Functioning (ICF Checklist for Oral Health). ${ }^{22}$

Clinical examination included: 1) report of pain by the patient and /or caregiver, and targeted examination of potentially painful teeth; 2) presence of dental plaque, assessed according to the criteria of Greene and Vermillion and reported using the Simplified Oral Hygiene Index (S-OHI); ${ }^{23}$ 3) gingival bleeding, measured on buccal and lingual surfaces of all teeth according to the criteria of Ainamo and Bay ${ }^{24}$ and reported using the gingival bleeding index $(\mathrm{GBI})^{24}$ and; 4) dental caries with a dmft or DMFT score according to the criteria of the World Health Organization $(\mathrm{WHO}){ }^{25}$

\section{Inclusion criteria}

Patients with a recognised disability and at least one dentinal carious lesion in a primary or permanent tooth without pulpal involvement, spontaneous pain or tooth mobility, but in occlusion with the antagonist tooth or teeth and in contact with the neighbouring tooth or teeth, were included in this clinical trial. Informed written consent was required for all participants. Consent was obtained from the patient him- or herself, if adult and cognitively able to consent, or from the patient's parent or legal guardian. Patients who could not be included in the study received appropriate dental treatment in the clinic or under GA according to clinical need.

\section{Attribution to treatment group}

At the initial visit, the study aims and design were explained to the patients and/or the parents or caregivers (hitherto referred to as 'respondents') of all those eligible for inclusion. Information was addressed to both patient and caregiver so that the patient with intellectual disability could be supported in the decision making process. Although caregiver's perceptions cannot be identical to patient's perceptions, none of the people included in the study had the mental capacity to give an independent informed decision, although their opinion was always sought. The need for treatment and the presence of carious lesions were explained and the respondents were informed of two treatment options, both verbally by the dentist, and with the use of printed brochures.

The brochures had undergone content validation at a meeting of a national Association for Disability and Oral Health and had been independently piloted in a special care clinic with respondents not participating in the current study. Respondents kept the brochures to read at home with the following given options: 1) Conventional treatment using rotary instruments, with or without local anaesthesia as required, and placement of composite resin under rubber dam (CRT). This treatment could be provided in the clinic or under general anaesthesia (GA). 2) ART approach ${ }^{26,27}$ using hand instruments only, with or without local anaesthesia as required, and placement of a restorative high-viscosity glass-ionomer.

At the second visit, respondents confirmed their choice of either ART treatment or conventional treatment and provided the written informed consent necessary for inclusion in the study.

\section{Treatment provided}

One of two operators commenced dental treatment using the approach selected by the respondent. This led to the following situations: 1) The selected treatment could be performed to an optimal clinical standard. If other restorations needed to be undertaken, further appointments were scheduled for treatment with the same technique. 2) The selected treatment could 
not be performed adequately because of lack of patient cooperation. If the selected treatment was the conventional approach, ART treatment was subsequently proposed. 3) Neither conventional treatment nor ART could be performed and the patient was referred for conventional restorative treatment under general anaesthesia (GA).

At this, and all subsequent appointments, a record card was completed with regard to tooth number, cavity type, treatment technique used, need for local anaesthesia, whether the treatment was carried out to an optimal clinical standard, the difficulties experienced during treatment, and the reasons for changing the treatment option if appropriate.

\section{Outcome measures}

Acceptability of the treatment was inferred from the restorative treatment selected by the respondents at baseline and the reasons recorded for choosing a given treatment.

Feasibility of the treatment was assessed by recording 1) whether the treatment was provided following optimal clinical standards; 2) whether one or more difficulties were encountered during treatment; 3) whether the treatment of choice could be provided; 4) the reasons for changing treatment approach; 5) the need for use of local anaesthesia.

Satisfaction with the treatment provided was assessed by scoring on a 10-point visual analogue scale, completed by respondents at 12 months. Respondents were asked to fill in a "Treatment Satisfaction Form" containing two questions: 1) Was the treatment carried out according to your expectations? and 2) After one year, has the treatment solved the problem of the patient's teeth? Each question was replied using 10-points scores of the scales, ranging from the lowest values related to negative perspectives up to the highest values that expressed a positive experience. A mean value of the two responses was quoted as a final satisfaction score using the following criteria: $0=$ total dissatisfaction to $10=$ complete satisfaction .

\section{Statistical analysis}

Data were entered into Excel ${ }^{\circledR}$ and analysis was undertaken by a biostatistician from the Dental School. ANOVA with Bonferroni correction and Chi-square tests were used to test for differences between the dependent variables, level of satisfaction, level of acceptability, level of feasibility and the independent variables, age, gender, treatment group and medical diagnosis. Some tests could not be performed because of too low a number of patients per cell. Statistical significance was set at $\alpha=0.05$.

\section{Results}

\section{Study population}

A total number of 66 patients were included in the study with a mean age of 13.6 years $(\mathrm{SD} \pm 7.8$; min. 3; max. 39 years). Thirty-six patients were male $(59 \%)$ and thirty patients were female (41\%). The most common principal medical diagnosis was Cerebral Palsy (39\%), followed by Autistic Spectrum Disorder $(20 \%)$, West syndrome (9\%), Down syndrome $(6 \%)$, Mental Retardation of unspecified origin (6\%) and Rett syndrome (5\%). Mean DMFT and dmft were $17.3 \pm 11.9$ and $7.8 \pm 8.6$, respectively. Prevalence of plaque and gingival bleeding was 100 percent. In all, 298 carious lesions were restored, 182 with ART and 116 with conventional treatment (of which 95 were restored under GA). ${ }^{21}$

\section{Acceptability of the treatments}

The ART approach was chosen at baseline by 43 respondents $(73 \%)$. The main reasons reported for selecting ART were problems coping with noise and vibration of the drill (51\%), and/or desire to avoid the drill (33\%). Respondents in this group also cited poor cooperation $(23 \%)$ and a desire to avoid a GA $(16 \%)$ as a reason for choosing a less invasive approach.

23 respondents selected the conventional approach, and 8 of these patients were programmed directly for treatment under GA. Those who selected a conventional approach in the chair stated that they preferred a recognised technique (33\%), and that they considered conventional treatment to be of a higher standard (33\%). Some were confident of cooperation as the patient had previous experience of treatment and had been able to cope (27\%). The 8 respondents who chose conventional treatment under GA reported that dental examination was impossible 
$(n=3)$, presence of pain or infection $(n=6)$, and/or that other treatment was also required in addition to the restorative treatment $(n=6)$.

Age was not statistically related to treatment option selected $(p=0.34)$. Respondents did not select GA for any female patients and 24 out of 26 respondents for patients with cerebral palsy selected ART. The pathways taken between selected treatment and treatment provided is illustrated in Figure.

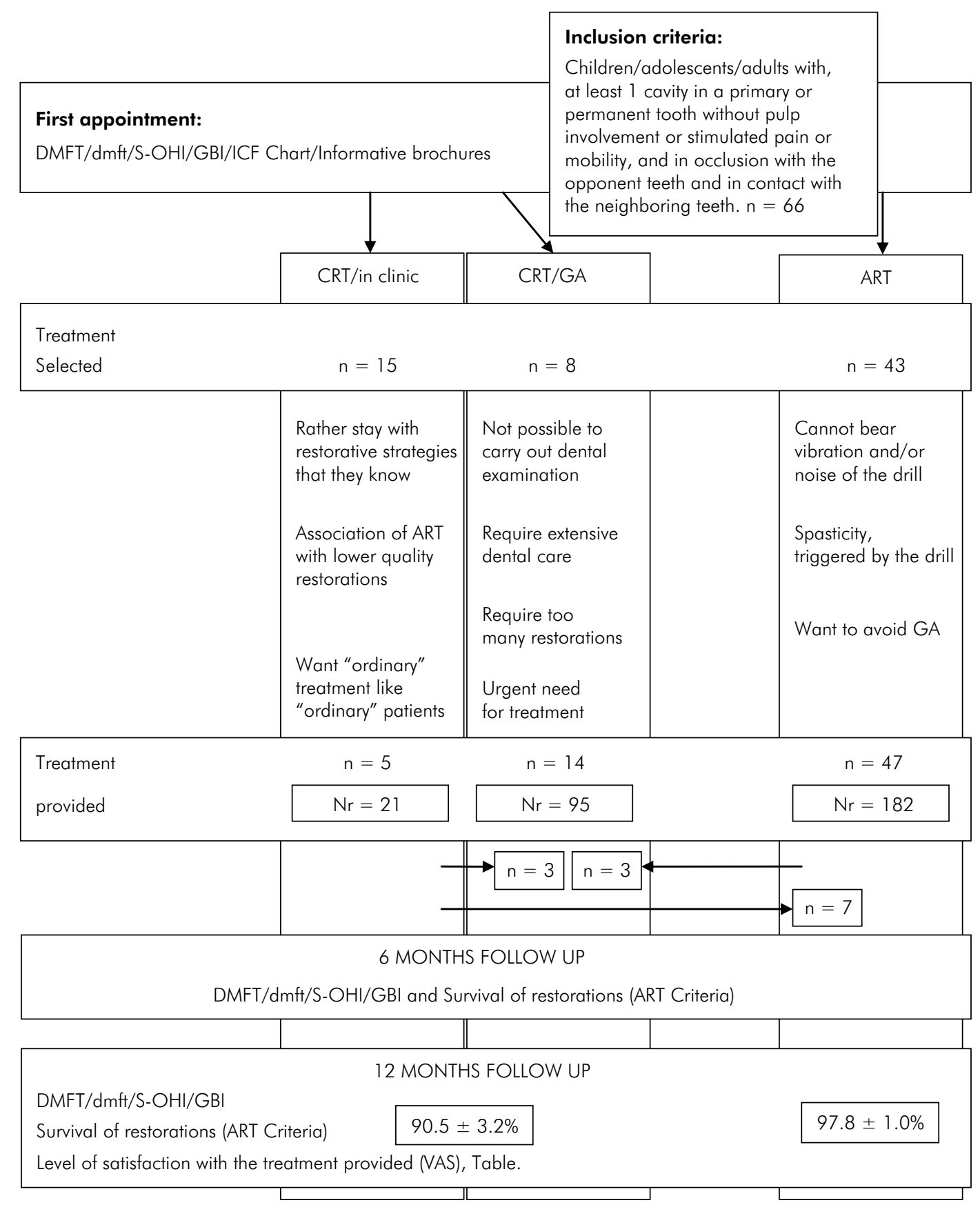

References: ART: Atraumatic restorative treatment; CRT: Conventional restorative treatment; n: Number of patients; Nr: Number of restorations; DMFT/dmft: Decayed, missing and filled teeth; S-OHI: Simplified oral hygiene index; GBI: Gingival bleeding index; ICF: International classification of functioning, disability and health; VAS: Visual analogue scale.

Figure. Flow chart of the selection and provision of different treatment options by treatment group and evaluation period of the study group. 


\section{Feasibility}

Optimal clinical standards for placement of restorations were obtained for 37 of the 47 patients who received ART treatment (79\%). Three out of five patients receiving conventional treatment in the clinic had restorations placed under optimal conditions and all of those undergoing conventional treatment under GA. Moisture control was cited as the main source of difficulty for those patients for whom restoration placement was not optimal.

Difficulties in providing restorations for the 52 patients whom received treatment in the clinic were noted, regardless of the ability to provide optimal treatment. Main problems included spasticity and uncontrolled movements (33\%), patient cooperation $(29 \%)$ and moisture control $(17 \%)$. Treatment presented no problem for only four patients. Moisture control was cited as the main source of difficulty $(n=7)$ for the 12 patients for whom restoration placement was not optimal, followed by lack of cooperation $(n=4)$.

Seven patients in the conventional treatment group could not have their treatment of choice at the first treatment attempt, as they were unable to cope with the drill $(n=6)$ or unable to cooperate $(n=1)$. All seven of these patients went on to receive treatment successfully, and with optimal restoration placement, using the ART approach. Three patients in the conventional treatment group were moved to the GA group following the first treatment attempt because of the quantity of treatment needed, a need for urgent treatment of infection or because restorative treatment was not the only treatment required. No patient moved from the ART to the CRT in the clinic group. Three patients in the ART group were moved to the GA group following the first treatment attempt. Reasons given were an inability to provide restorations of optimal standard $(n=2)$ and difficulty cooperating due to hyperactivity.

Local anaesthesia was required for four of the 47 patients receiving ART treatment and for three of the five patients receiving conventional treatment at the chair. All patients undergoing GA received local anaesthesia. Age was not statistically related to the type of treatment provided $(p=0.36)$. Ten males and 4 females were treated under general anaesthesia, and only females were treated with CRT in the clinic $(n=5)$.

\section{Satisfaction with the treatment techniques}

The level of satisfaction reported by the respondents was highest in the group that had selected, and subsequently received, ART (mean VAS score 9.2 \pm 0.9 ), followed by those selecting and receiving conventional treatment under GA (mean VAS and SD score: $8.1 \pm 1.1$ ) and those selecting, and receiving conventional treatment in the clinic (mean VAS and SD score: $7.4 \pm 1.1$ ). Respondent satisfaction was statistically significantly higher for those receiving ART than for those receiving CRT in both the clinic and under general anaesthesia (Bonferroni; $a=0.05$ ). Full satisfaction results are shown in Table.

\section{Discussion}

\section{Methodological aspects}

The current study does present a number of limitations. The study size was small in terms of numbers of patients, despite the 6 months inclusion period. A small number reduces the power of a study and becomes problematic in analytical studies but not so much in a descriptive study as the present one. Nevertheless, the present study might best be considered a pilot study. In addition, respondents in the

Table. Selected and provided treatment, and the level of satisfaction of patients/caregivers at 1 year follow up.

\begin{tabular}{|c|c|c|c|c|}
\hline \multirow{2}{*}{$\begin{array}{l}\text { Treatment Selected } \\
\text { Frequency } \\
\text { Percentage } \\
\text { Mean level of satisfaction } \pm \text { SD }\end{array}$} & \multicolumn{4}{|c|}{ Treatment Provided } \\
\hline & ART & $\begin{array}{l}\text { CRT/in } \\
\text { clinic }\end{array}$ & CRT/GA & Total \\
\hline \multirow[t]{3}{*}{ ART } & 40 & 0 & 3 & 43 \\
\hline & 60.6 & 0.00 & 4.6 & 65.2 \\
\hline & $9.2 \pm 0.8$ & & $7.6 \pm 0.6$ & \\
\hline \multirow[t]{3}{*}{ CRT/in clinic } & 7 & 5 & 3 & 15 \\
\hline & 10.6 & 7.6 & 4.6 & 22.7 \\
\hline & $8.8 \pm 0.9$ & $7.4 \pm 1.1$ & $7.6 \pm 1.1$ & \\
\hline \multirow[t]{3}{*}{ CRT/GA } & 0 & 0 & 8 & 8 \\
\hline & 0.00 & 0.00 & 12.1 & 12.1 \\
\hline & & & 8. $4 \pm 1.2$ & \\
\hline \multirow[t]{2}{*}{ Total } & 47 & 5 & 14 & 66 \\
\hline & 71.2 & 7.6 & 21.2 & 100 \\
\hline
\end{tabular}

ART: Atraumatic restorative treatment; CRT: Conventional restorative treatment; GA: General anaesthesia; SD: Standard deviation. 
study represented both patients and their caregivers, which raised the problem of proxy perception of treatment and satisfaction. The attribution of patients to each treatment group could not be randomised given the nature of the study population but a second best alternative was used by asking the respondents to select a treatment option, rather than the dentist.

By doing so, it was possible to follow a comprehensive framework for conceptualising the quality of the service provided by searching the reasons for choosing one or the other procedure, exposing expectations with regards the options that were offered, and receiving feedback on level of satisfaction with the service received..$^{28}$ Quality is a complex concept and the definition of good quality often depends on the person defining it. However, for policy makers, health providers and managers, it is important to measure the quality of health strategies in order to develop better programmes, not only for improving access to care but also to meet the needs of the population with effective solutions. ${ }^{28}$

Visual analogue scales, are commonly used in quality research in Health Science to assess subjective variables, such as pain. Such tools are valuable in the investigation of domains such as patient expectations and level of satisfaction in clinical studies, and have been previously used as proxy measures. ${ }^{29}$ In this study, only an absolute scale was feasible as respondents could not compare their level of satisfaction with regards different treatment modalities of which they had no experience. Inclusion of such measures offers a relatively holistic understanding of how different therapeutic strategies impact on the well-being of those participating in a clinical study.

\section{Main findings}

The results of the present study show that ART appears to be a more satisfying, feasible, acceptable and effective approach to restorative treatment in patients with disability than the conventional restorative treatment.

In terms of acceptability, ART was the approach of choice at baseline for patients and/or their caregivers, and it gave the highest level of satisfaction post-treatment, whether it had initially been selected or not. The main perceived advantage of this approach was avoidance of the dental drill, which is associated with noise, vibration and water spray. All of these stimuli may be difficult to cope with for both persons with anxiety disorders and persons with functional disability, such as spastic movement or swallowing difficulties. Another advantage of the ART approach is that it is rarely necessary to use local anaesthesia and therefore the patient avoids the anxiety related to needles and injections. In the present study, only four patients required local anaesthesia for ART treatment. A final advantage perceived by patients and caregivers was the potential to avoid an intervention under general anaesthesia. This aspect of ART is particularly significant for persons with disability who often present multiple medical diagnoses and are at higher risk of the problems of morbidity and mortality associated with GA. ${ }^{15}$ In addition, it makes the technique attractive in terms of improved accessibility to general dental services, thus reducing the health costs associated with the treatment of this population.

It is interesting to find that the 23 respondents who selected the conventional approach stated that they would rather stick to better-known alternatives, relating ART to lower quality treatment standards. This point of view was expressed in a previous survey of special care dentists' attitudes to ART, with $30 \%$ of respondents perceiving ART as 'lower quality dentistry'. ${ }^{20}$ Five respondents went as far as to say that they would probably not use ART even if 'reliable scientific evidence showed the suitability and effectiveness of the ART approach in this population'. It is hoped that the present results related to acceptance of ART as well as those confirming a higher survival rate for ART than conventional restorations at 12 months in this population, ${ }^{21}$ may help to dispel the persistent mistrust of ART amongst professionals and encourage them to add this approach to their therapeutic arsenal.

The results of the current study show that not only was ART treatment more feasible in the clinic than conventional treatment but that a higher proportion of ART restorations could be placed under optimal conditions. This finding is extremely important when taken in parallel with the types of disability presented by the study population and also the difficulties 
reported by the dental practitioners during treatment. It would seem that ART proves to be a more effective solution for patients unable to cope with conventional treatment in the clinic, even in service conditions where adjunctive management techniques (such as conscious sedation) are present. In addition, previous authors have suggested that positive behavioural changes towards future dental care may be observed in patients with intellectual disabilities following use of minimally invasive techniques. ${ }^{30}$

The ART approach clearly has the potential to greatly improve patient experience of dental treatment and to improve access to restorative treatment in the clinic. It could also be speculated that health costs and patient morbidity might be reduced by diminishing referrals for GA, although further research is necessary to quantify the potential benefits.

\section{Conclusion}

This study is the first of its kind in the population with disability and it confirms the acceptability and the feasibility of the use of the ART approach for these

\section{References}

1. World Health Organisation. World Report on Disability, 2011. Geneva (Switzerland): WHO Press; 2011.

2. Nunn J, Freeman R, Anderson E, Carneiro LC, Carneiro MS, Formicola A, et al. Inequalities in access to education and healthcare. Eur J Dent Educ. 2008 Feb;12 Suppl 1:30-9.

3. Leroy R, Declerck D. Oral health care utilization in children with disabilities. Clin Oral Investig. 2013 Nov;17(8):1855-61.

4. Christensen LB, Hede B, Petersen PE. Public dental health care for persons with disability. Acta OdontolScand. 2005Oct;63(5):278-83.

5. Anders PL, Davis EL. Oral health of patients with intellectual disabilities: a systematic review. Spec Care Dentist. 2010 May-Jun;30(3):110-117.

6. Collado V, Nicolas E, Hennequin M. Dental difficulty for adult patients undergoing different dental procedures according to level of dental anxiety. Odontostomatol Trop. 2008 Dec;31(124):35-42.

7. O'donnell D. The special needs patient. treatment in general dental practice: is it feasible?. Int Dent J. 1996 Aug;46(4):315-9.

8. Stiefel DJ, Truelove EL, Mandel LS. Perceived barriers vs dental care availability for persons with disabilities. J Dent Res. 1991(Spec issue);70:337.

9. Gordon SM, Dionne RA, Snyder J. Dental fear and anxiety as a barrier to accessing oral health care among patients patient groups. However, larger, long-term studies are needed in order to build a stronger evidence base to confirm or refute the positive benefits of the ART approach for persons with disability suggested by the current results.

\section{Acknowledgements}

Authors are very grateful to Drs. Ricardo Cabral, Laura Brain and Claudia Bonnin (The Dental Faculty, Universidad Nacional de Córdoba, Argentina) for their assistance in implementing this clinical trial, and to Jan Mulder (Global Oral Health Department, Radboud University Medical Centre, Nijmegen, The Netherlands) for analysing the data. We thank Dentsply, Germany and GC Europe, Belgium for supplying the glass-ionomers and 3M ESPE, Argentina, for supplying the adhesive system and resin composite. The study was co-financed by institutional funds from the SECYT, Universidad Nacional de Córdoba (Argentina) and the Global Oral Health Department, Radboud University Medical Centre, Nijmegen (The Netherlands).

with special health care needs. Spec Care Dentist. 1998 Mar-Apr;18(2):88-92.

10. Cumella S, Ransford N, Lyons J, Burnham H. Needs for oral care among people with intellectual disability not in contact with the community dental services. J Intellect Disabil Res. 2000 Feb;44(Pt1):45-52.

11. West GA, Reid KH, Bastawi AE. Autonomic responses to dental procedures in pedodontic patients during a standard restoration session. J Dent Res. 1983 Jun;62(6):728-32.

12. Molina G, Kultje C. Atraumatic Restorative Treatment with Carisolv in intellectually disabled patients: 1 year follow-up. J Disabil Oral Health. 2003 Apr;4(1):15-8.

13. Hussein I, Kershaw AE, Tahmassebi JF, Fayle SA. The management of drooling in children with mental and physical disabilities. A literature review. Int J Paediatr Dent. 1998 Mar;8(1):3-11.

14. Tsai CL, Tsai YL, Lin YT, Lin YT. A retrospective study of dental treatment under general anesthesia of children with or without a chronic illness and/or a disability. Chang Gung Med J. 2006 Jul-Aug;29(4):412-8.

15. Lee HH, Milgrom P, Starks H, Burke W. Trends in death associated with pediatric dental sedation and general anesthesia. Paediatr Anaesth. 2013 Aug;23(8):741-6. 
16. Molina GF, Leal S, Frencken JE. Strategies for managing carious lesions in patients with disabilities: a systematic review. J Disabil Oral Health. 2011 Nov;12(4):159-67.

17. Carvalho TS, Ribeiro TR, Bönecker M, Pinheiro EC, Colares V. The Atraumatic Restorative Treatment approach: an "atraumatic" alternative. Med Oral Patol Oral Cir Bucal. 2009;14:668-73.

18. Frencken JE, LealSC, Navarro MF. Twenty-five-year Atraumatic Restorative Treatment (ART) approach: a comprehensive overview. Clin Oral Investig. 2012 Dec;16(4):1337-46.

19. Schriks MC, van Amerongen WE. Atraumatic perspectives of ART: psychological and physiological aspects of treatment with and without rotary instruments. Community Dent Oral Epidemiol. 2003 Feb;31(1):15-20.

20. Molina GF, Faulks D, Frencken JE. Suitability of ART approach for managing caries lesions in people with disability: experts' opinion. Acta Odontol Scand. 2013 Nov;71(6):1430-5.

21. Molina GF, Faulks D, Mazzola I, Mulder J, Frencken JE. One year survival of ART and conventional restorations in patients with disability. BMC Oral Health. 2014 May 7;14:49. doi: 10.1186/1472-6831-14-49.

22. Faulks D, Norderyd J, Molina G, Macgiolla Phadraig C, Scagnet G, Eschevins C, et al. Using the international classification of functioning, disability and health (ICF) to describe children referred to special care or paediatric dental services. PLos One. 2013 Apr 16;8(4):e61993. doi: 10.1371/journal.pone.0061993.

23. Greene JC, Vermillion JR. The simplified oral hygiene index. J Am Dent Assoc. 1964 Jan;68(1):7-13.

24. Ainamo J, Bay I. Problems and proposals for recording gingivitis and plaque. Int Dent J. 1975 Dec;25(4):229-35.

25. World Health Organisation. Oral health surveys, basic methods. 4th Geneva: World Health Organization; 1997.

26. Frencken JE, Leal SC. The correct use of the ART approach. J Appl Oral Sci. 2010 Jan-Feb;18(1):1-4.

27. Frencken JE, Holmgren CJ. Atraumatic Restorative Treatment (ART) for dental caries. 1st ed. Nijmegen; 1999.

28. Lawthers AG, Pransky GS, Peterson LE, Himmelstein JH. Rethinking quality in the context of persons with disability. Int J Qual Health Care. 2003 Aug;15(4):287-99.

29. Mcgregor AH, Doré CJ, Morris TP. An exploration of patients' expectation of and satisfaction with surgical outcome. Eur Spine J. 2013 Dec;22(12):-2836-44.

30. Guare R, Ciamponi AL, Romano MM. Behavioral and physiological changes in children with Down syndrome using mechanical and chemomechanical (Carisolv) caries removal methods. Spec Care Dentist. 2008 Sep-Oct;28(5):195-200. 